BIO Web of Conferences 1, 00083 (2011)

DOI: $10.1051 /$ bioconf $/ 20110100083$

(C) Owned by the authors, published by EDP Sciences, 2011

\title{
Evaluation of multimodal feedback effects on improving rowing competencies
}

\author{
Yifat Shorr $^{*}$ Alessandro Filippeschi ${ }^{\dagger}$ Daniel Gopher ${ }^{*}$ Emanuele Ruffaldi ${ }^{\dagger}$ Maria Korman ${ }^{*}$
}

\author{
(*)Technion,Haifa,Israel \\ (†)Scuola Superiore S.Anna, Pisa, Italy \\ E-mail:yifat.shorr@gmail.com,a.filippeschi@sssup.it,dgopher@ie.technion.ac.il, \\ e.ruffaldi@sssup.it,korman.maria@gmail.com
}

\begin{abstract}
This study focused on the selection and preliminary evaluation of different types of modal and information feedback in virtual environment to facilitate acquisition and transfer of a complex motor-cognitive skill of rowing. Specifically, we addressed the effectiveness of immediate information feedback provided visually as compared to sensory haptic feedback on the improvement in hands kinematics and changes in cognitive load during the course of learning the basic rowing technique. Several pilot experiments described in this report lead to the evaluation and optimization of the training protocol, to enhance facilitatory effects of adding visual and haptic feedback during training.
\end{abstract}

\section{Introduction}

Skills are gradually acquired with experience and practice time [2, 7]. Motor skills, such as rowing, require coordinating motion efforts, motor-cognitive skills, body strength, extensive practice, close guidance and coaching to achieve high competency. Novices are characterized by poor and slow skill performance, effortful and under a close conscious supervision. Relevant information is missed or misinterpreted; redundancies and higher level structures are not recognized and not used, elements of procedures are skipped or not performed in order. These problems are reduced or completely disappear in expert performance where procedures are performed rapidly, smoothly, as a unified response unit, requiring little conscious supervision with limited conscious supervision (automatic), and making use of higher level structures and redundancies [4]. A multimodal virtual reality (VR) environment offers many degrees of freedom which enrich the traditional supervision and guidance of the coach enabling performance to become more objectively evaluated and communicative to the trainee. The on-line information can be given as a multimodal feedback that spans the senses and includes visual, auditory, and haptic/kinematic interactions [12]. It has been found [8] that augmented feedbacks greatly improve training of highly complex patterns of bimanual coordination and postural control as can be found in rowing. These feedbacks assist the coach in generalizing the global organization of interacting local (e.g., muscular, articular, perceptual) components [1]. Augmented feedbacks play a dual role in the learning process: first, by providing the learner with performance information about the success of the movement in progress or what must be done on a succeeding performance attempt; second, by motivating the learner to continue striving toward a goal [10]. Augmented feedbacks can be given in different forms: knowledge of result (KR) informs the performer on the match between his action and the designated target or goal; knowledge of performance (KP) gives indication about movement characteristics e.g. the left arm was held in perfect height or the hand was not stable; augmented sensory feedback means that an external device is used to enhance sensory feedback. Multiple-resource-theory of attention states that a task that engaged different forms of input modalities will be performed better than tasks completed within the same modality [10]. The use of more than one modality can improve perception and enhance performance and as well affect the cognitive load. Kahneman [6] has shown that pupil size can serve as an index of processing load: pupil's dilation correlates with increasing cognitive workload. In a system with complex multiple stimulations the attention load may exceed processing capacity and one task element or another must suffer. Since attention management under high load can be improved with training [5], we assume that during practice, the participant acquires distribution strategy to all the tasks. We expect that training will induce quantitative and qualitative

This is an Open Access article distributed under the terms of the Creative Commons Attribution-Noncommercial License 3.0, which permits unrestricted use, distribution, and reproduction in any noncommercial medium, provided the original work is properly cited. 
changes in kinematics, coordination (timing) and cognitive load: 1) accuracy of hands movement geometry will increase as practice continues;2) coarticulation will develop inducing smoother trajectory with higher velocitiesat reference points as $A B C$ in later discussion; 3) timing of rowing cycle will improve; 4) cognitive load will decrease.

\section{Method}

Rowing is a complex task involving physical and cognitive skills, coordination between body organs, respiratory system and pace. We adopt a simplified rowing task that requires production of smooth and cyclic hands movement between 3 points $\mathrm{A}, \mathrm{B}$ and $\mathrm{C}$ in 2D plane, representing the three critical phases of hand's movement during the stroke: catch, finish and start of recovery, respectively. The time sequence of the points is shown in Fig. 1.

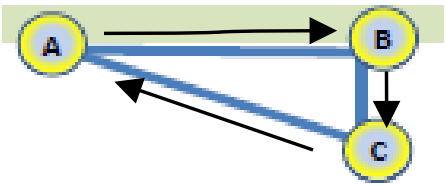

Fig. 1 Geometrical representation of hands trajectory during rowing cycle comprised of coordinated movement between the 3 points A,B and $\mathrm{C}$. Point A corresponds to the initial point of the catch phase. Points B and C are near the participant's chest, correspond to finish and start of recovery, respectively. The errors indicate the direction of correct movement.

Participants are male adults screened for handedness and general health (BMI), with no prior experience in rowing. They are asked to maintain regular sleep schedule during the experiment. All participants sign informed consent.

The system adopted is an instantiation of the SKILLS Lightweight Rowing Platform that includes: Concept 2 ergometer device; LCD screen; two Vicon motion capture cameras for tracking ergomenter's handle; an eye tracker; airflow haptic system and a metronome.

Two components comprised the visual feedback on the LCD screen. The first is the display of Virtual Environment showing the rowing scenario with participant's boat, moving according to the speed computed by the ergometer. The second component is the representation of the three critical points. Three bars, corresponding to the position of the ergometer handle at the three critical points of the rowing cycle, are overlaid either on the rowing scenario or on the black screen. When feedback is enabled the bars are dynamically updated at the end of each cycle during the recovery phase for about 3 seconds. The bars are colored in green, if a given target point was reached, or red with a yellow line if it was missed, indicating the direction of the mistake. When this feedback is not enabled, the bars are statically shown on screen in yellow. In addition, for supporting the eye tracking, a fixation cross is constantly shown in the center of the screen.

In the haptic feedback condition flexible plastic tubes are positioned along the plane of rowing movements to produce the desired air pressure on the outer part of participant's hand at the catch (A) position and the finish (C) position. In this way the subject perception of the air-flow is related to the correctness of the movement. This haptic feedback is based on TAEMA CP90 (Taema; France), which is a conventional continuous positive airway pressure device (CPAP).

In total there are eight possible combination of feedback, that have been combined to form three different training conditions as shown in Table 1 at the end of the paper. First the no feedback condition, that will be used by the control group, then a haptic condition with the airflow, black screen and static yellow bars, and finally a visual condition with the dynamic rowing environment and augmented feedback provided by the three bars.

Participants were asked to train on a repetitive rowing movement by moving through the three target points repeatedly and to maintain the low stroke pace of 15 SPM, as indicated by the metronome, and subject to the minimum resistance in the ergometer settings. At each metronome tick the participant should start a new stroke. Participants are trained for 4 sessions, spaced 13 days apart, and then tested for retention and transfer in a fifth session. Each session is comprised of a PreTest block with externally paced rowing movement without any feedback, and Training blocks. During training blocks, the participant is either provided (experimental groups) or not (control group) with an immediate feedback on their performance (see Table 1). All training blocks were performed at a constant low stroke rate (15 SPM) and at minimum resistance. During the fifth session, Pre-Test and Transfer blocks were performed with change of resistance, rowing pace, and targets order by reversing the temporal sequence of the ABC points. During the course of this multi-session learning protocol, participants are expected to improve their performance differentially, according to the type of feedback that was afforded in the blocks. Eye-tracker measurements were taken only during training blocks, with the purpose of analyzing attentional effects during training.

\section{Preliminary Results}

In the preliminary phase of this experiment we tested two subjects: one trained in Visual feedback condition and another trained in Haptic feedback condition. Figure 2 depicts the evolution of their hand 
trajectories. In Visual condition, on day one pre-test, the three targets were consistently missed. Moreover,

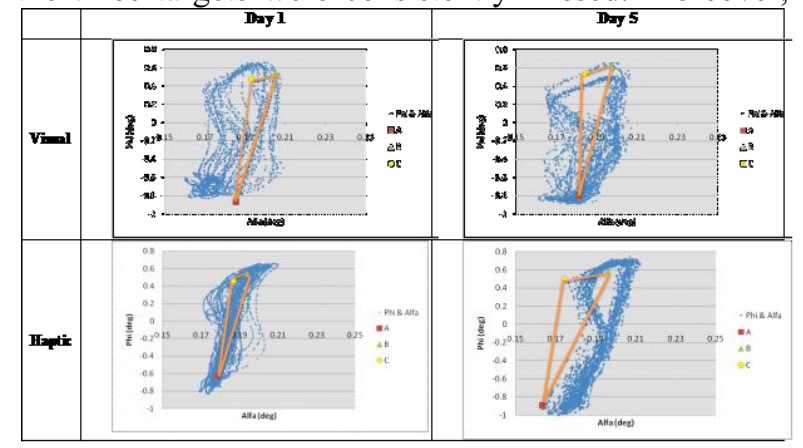

Fig. 2. Trajectories (superimposed) at tests at the beginning and at the end of training. Data for two participants are shown. Alfa and Phi angles are related to the changes in hands position on $\mathrm{X}, \mathrm{Y}$ axes respectively, being the equivalent of the oar angles in the full rowing task. Colored dots represent the target position of $\mathrm{A}, \mathrm{B}$ and $\mathrm{C}$ points. The movement trajectory becomes smoother and the variance is reduced.

the overall shape of the trajectory, that should resemble a rain drop shape when the task is skilled, is jerky. On day five retention session the trajectory shape has been significantly changed: it got a clear drop shape on all strokes while the accuracy of going through the A, B and $\mathrm{C}$ points was significantly increased. Trajectory changes were accompanied by an increase in mean speed at the three target points. These observations are in line the predictions of the minimum jerk model [3]. In particular the minimum jerk model assumes that given a starting point, an end-point and one or more via-points, that are identified as the position in the path where a local minimum speed is attained, corresponding to the point of local maximum curvature, the motor system preplans an entire hand trajectory that passes through all these points with the smoothest possible (i.e. minimum jerk) trajectory, implying increase in the angular velocity at via points.

On the meanwhile, the subject that was trained in haptic condition shows a different pattern of results. On day one pre-test, he performed relatively well with regard to the accuracy of passing through the points $A$, $\mathrm{B}$ and $\mathrm{C}$. However, in terms of movements' geometry, his performance was very poor, jerky and composed of discrete segmented movements. Training induced significant improvement in the overall shape of the path and there was an increase in the angular movement velocities at target points. However, the accuracy of passing through the points was decreased. This suggests that speed-accuracy trade-off characterized the course of training, as opposed to the results of the first subject.

Regarding attention along the course of training, pupil diameter decreased (data for one subject is shown in Fig. 3), suggesting that changes in attentional load are correlated with the acquisition of the rowing skill.

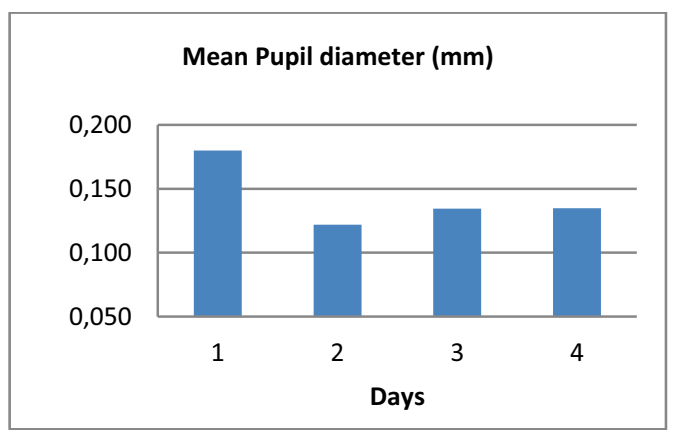

Fig. 3. Mean pupil diameter at different training sessions. Data of a single subject in visual only feedback training condition is shown. Decrease and stabilization of the Pupil diameter are apparent with the progress of multi-session training.

\section{Conclusions}

The experimental protocol, although in the preliminary stage of subject testing, has already presented interesting possibilities in training coarticulation in complex motor schemes. While the overall scheme seems promising at this stage no conclusions can be made when comparing the effect of different feedback types.

\section{Acknowledgments}

This work was supported by the SKILLS Integrated Project (IST-FP6 \#035005, http://www.skills-ip.eu) funded by the European Commission.

\section{References}

[1] Faugloire E., Bardy BG., Stoffregen TA., Dynamics of Learning New Postural Patterns: Influence on Preexisting Spontaneous Behaviors : Journal of Motor Behavior. Volume 38, Number 4 / July 2006 299-312

[2] Fitts, PM (1964). Perceptual-motor skill learning. In AW Melton (Ed.), Categories of human learning (pp. 243-285). NY Academic press.

[3] Flash T, Hogan N (1985) The coordination of arm movements: an experimentally confirmed mathematical model. J Neurosci 5(7):1688-1703

[4] Ericsson, K. Anders; Krampe, Ralf T.: The role of deliberate practice in the acquisition of expert performance. Psychological Review, Vol 100(3), Jul 1993, 363-406

[5] Gopher, D. (1993). The skill of attention control: Acquisition and execution of attention strategies. In D. Meyer \& S. Kornblum (Eds.). Attention and Performance XIV: Synergies in Experimental Psychology, Artificial Intelligence, and Cognitive Neuroscience - A Silver Jubilee. Cambridge, MA: MIT Press.

[6] Kahneman,D. J. Beatty, 1966: Change in cognitive load affects pupil diameter (PD), fixation duration and number of blinking

[7] Karni, A., Meyer, G., Jezzard, P., Adams, M., Turner, R., \& Ungerleider, L. G. (1995): 
Functional MRI evidence for adult motor cortex plasticity during motor skill. Learning. Nature, 377, 155-158.

[8] Kelso, S. (1995). Dynamic Patterns: The selforganization of brain and behavior. MIT Press, Cambridge, MA.

[9] Swinnen SP, Jardin K and Meulenbroek R, Between-limb asynchronies during bimanual coordination: Effects of manual dominance and attentional cueing; Neuropsychologia Volume 34, Issue 12, December 1996, Pages 1203-1213

[10] Magill, R. A. (1993) Motor learning: Concepts and Applications, $4^{\text {th }}$ ed. Brown

\begin{tabular}{ll}
\hline Feedback & Description \\
Sone (control) & $\begin{array}{l}\text { Static black screen and static yellow bars. No } \\
\text { haptic feedback is provided. } \\
\text { Dynamic rowing scenario and static yellow } \\
\text { bars. Haptic feedback is provided for A and C } \\
\text { locations. }\end{array}$ \\
Visual & $\begin{array}{l}\text { Dynamic rowing scenario with dynamic bars on } \\
\text { recovery phase. The bars represent on screen the } \\
\text { same spatial organization of the phases in stroke } \\
\text { (catch, finish, start of recovery). At the end of } \\
\text { each stroke bars' colors are updated to indicate } \\
\text { overall accuracy of movement in the ABC part. } \\
\text { Direction of error is also shown. }\end{array}$
\end{tabular}

Table 1. Experimental groups and the corresponding layout of the training environment.
[11] Wickens, C D.; Vidulich M, Sandry-Garza D, Principles of S-C-R Compatibility with Spatial and Verbal Tasks, The Journal of the Human Factors and Ergonomics Society, Volume 26, Number 5, October 1984 , pp. 533-543(11)

[12] Ruffaldi E., Filippeschi A.,. Avizzano C. A,. Bardy B., Gopher D., Bergamasco M. Feedback, affordances, and accelerators for training sports in virtual environments. Presence: Teleoperators and Virtual Environments, 20:33-46, 2011 